\title{
AUTHENTICATION OF DIGITAL MP4 VIDEO RECORDINGS USING FILE CONTAINERS AND METADATA PROPERTIES
}

\author{
D.P. Gangwar \\ Head of Cyber Forensics \\ dpgangwar@yahoo.com \\ Anju Pathania \\ Senior Scientist \\ pathania.anju@gmail.com \\ ${ }^{1}$ Anand, ${ }^{2}$ Shivanshu \\ $1 \& 2$ Scientists, Central Forensic Science Laboratory, Chandigarh, India
}

\begin{abstract}
The authentication of digital video recording plays a very important role in forensic science as well as for other crime investigation purposes. The field of forensic examination of digital video is continuously facing new challenges. At present the authentication of the video is carried out on the basis of pixel-based analysis. Due to the change in technology, it was felt that a new approach is required for the authentication of digital video recordings. In the present work a new approach i.e. analysis of media Information and structural analysis of video containers (boxes/ atoms) of mp4 file format have been applied for identification of original and edited videos. This work is limited only for Mp4 file format because the MP4 compressed format is widely used in most of the mobile phone for video recording and transmission purposes. For this purpose, we recorded more than 200 video samples using more than 20 different mobile phones of different make and models and more than 12 video editors, which are available in open source used for editing purpose. The original and edited MP4 video files were analyzed for their different metadata and structural contents analysis of different file containers (boxes/atoms) using different freeware tools. The details of the work are described below.
\end{abstract}

KEYWORDS - File Containers, Atom, File Structure, Metadata, Codec.

\section{Introduction}

Video manipulation is now within the reach of a common person due to the easy availability of many video editing tools in the open-source platform. The manipulation in the video can be used in various purposes for political propaganda, revenge, porn and child-exploitation material etc. The present video authentication techniques are based entirely on the observations of anomalies in the pixel domain in the video. Due to advancement in the video editing technology and sometimes large number of recordings comes for the examination it becomes practically impossible for the forensic scientists to detect the manipulation in the videos on the basis of pixel or frame base analysis. Hence, it was felt that the new, fast and reliable methods are required beyond the pixel domain. To overcome these problems, we proposed a new technique based on analysis of metadata properties and file containers (boxes/atoms) of the MP4 video files for identification of original and edited video for authentication purpose.

\subsection{Metadata of the MP4 video Files}

The Digital video file contained a bunch of information hidden inside it. This hidden information is called the metadata properties of the digital file. The metadata includes file size, file format, video and audio codec details, DOC, DOM, device information, UTC date \& time, GOP information, picture resolution, bit rate, frame rate mode, bit depth, mvhd, audio codec ID, Sampling rate, picture resolution etc. as shown in Table-1. The mp4 format has its own specification of metadata as per standard ISO/IEC 14496-14:2003) and QuickTime File Format. Hence, whenever the original video recording edited using some editor its metadata would change as the video editor use different codec and writing library for editing and re-saving purpose. These changes in these metadata details are very useful signs to detect the editing in the original video recordings. 


\subsection{MP4 Containers (boxes/atoms) structures}

Nowadays the compressed MP4 video format is very common, as it supports several most used codec and as broadly supported by different purposes including transmission. The MP4 Container is mainly specified by ISO/IEC 14496-12, although some boxes are elaborated by ISO/IEC 14496-15 and ISO/IEC 14496-14. The MP4 container's (boxes/atoms) having a definite structure as shown in. Fig.1a, Fig.1b \& Fig.1c as per the ISS/IEC 14496-12:2005(E) and Apple Quick time the structure of file containers (atom/boxe) are arranged a defined order. These atoms contained further other atoms which called the children atoms. The each atoms contained specific information inside it. The MP4 file contained the definite atoms and the brand of mobile almost has the similar atoms and similar arrangement of the atom's structure. The MP4 video file format mainly contained three boxes i.e. "ftyp" (file type box), "mdat" (media data) and "moov" (related to all segments). The atom "ftyp" contained the details about major brand and minor version and the file size, header size, data size, begin and end position about the video. The second atom is "mdat" (media data box) contained media data of the file. The most important atom in mp4 files is "moov" which contained further many atoms of different names. The structure of the same is shown in Fig.1a , Fig1b \& Fig.c The "moov" atom is the heart of the file contained the atoms "mvhd"(movie header), "udta"(user data-contained device info and GPS location), "meta"(contained information about the original source) and audio \& Video "trak" atoms. The video "Trak" contained "tkhd" (track header), "mdia". The "mdia" further contained "mhhd"(media header), "hdlr"(handler description), "minf"(media information)-contained the "vmhd"(video media header), "dinf'(data information)contained "dref"(data reference box) and "stbl"(sample table box). The "stbl "further contained "stsd"(sample description box)-contained avc1 file, which further may contain three boxes i.e (avcc, pasp, colr). The box "stts"(Decoding time to sample box), "stss"(sync sample box), "stsz"(sample size box), "stsc"(sampleto chunk box), "stco"(chunk offset box). The audio "trak" box contained the similar structure of boxes as video but containing the audio information.

\section{RELATED WORK}

The online survey of the literature reveals that the authenticity of digital MP4 video recording using metadata and file containers are published limited work so for. In my previous work [1] \& [2] I had used the metadata properties and Codec analysis for image and audio authentication. M. Harran et al. [3] in his study have mentioned tools used for extraction the metadata of digital image file for authentication purpose. In the case of Digital Video Thomas Gloe et al [4] were analyzed of the structures of the videos of AVI and MP4 video streams of mobile phones and digital cameras. The authors use customized parsers to extract all the format structures of videos and report considerable differences in the choice of container formats, audio and video compression algorithms, acquisition parameters, and internal file structure. In combination, such characteristics can help to authenticate digital video files by distinguishing between original and post-processed videos. In his work "Forensic analysis of video file formats" had described the method for video container format of different camera and mobile phones to verify the authenticity of digital video streams. J. Randolph hall [5] in their master thesis "MPEG--4 video authentication using file structure and metadata" had utilized for comparison the original and edited digital video recording. Jieun Song et al. [6] in his work "Integrity verification of the ordered data structure in manipulated video contents" had proposed the method for detection the integrity of the video using the ordered data structure. David Gijeera et al. [7] in the work "We Need No Pixels: Video Manipulation Detection Using Stream Descriptors" mentioned that propose to identify forged videos by analyzing their multimedia stream descriptors with simple binary classifiers. RAQUEL RAMOS LÓPEZ et al. [8] in the work "We Need No Pixels: Video Manipulation Detection Using Stream Descriptors" mentioned that propose to identify forged videos by analyzing their multimedia stream descriptors with simple binary classifiers. BRIAN C. HOSLER et al. [9] Created the data bank of file's container structure of different camera and mobile phone and the same were utilized for source identification. Dasara Shullani et al. [10] stored the database of a large number of mobile and social media app and try to detect the source of audio and video recording. Massimo Iuliani et al. [11] in his work differentiated in the file container structure and contents introduced by different manufacturers, models and software processing. They also make a program which automatically detects the forgery in the video without video re-encoding. Carlos Quinto Huamán et al. [12] had studied different containers structure of smartphone video of different formats like mp4, 3gp and mov for identification of brand of capturing source, tracing the social networks like Facebook, YouTube, LinkedIn, Instagram, Twitter, Tumble, FaceBook Msn, Whatsapp, Telegram and identify the editing manipulation program used. 


\section{Proposed work}

The researchers so far had applied the meta-data and containers analysis for verification of integrity of video briefly. They have mentioned only basic details and structure of containers. They have not analyzed the details of contents inside the atoms/boxes. In the present study, Authors have taken a large sample size of different category more than 12 video editors software in three category i.e. installed video editing software, online video editing tool, Mobile app based tool. I have also used more than 20 different make and model of mobile phone for capturing MP4 videos. The both original and edited videos were analysis for metadata and file containers using different free ware tools available in open source. I have also analyzed the contents of the video containers(atoms/boxes) for better accuracy in video tempering detection.

\section{MATERIALS AND METHODS}

\subsection{Recorder i.e. Mobile Phone used to record original video samples}

The video samples were recorded in mp4 format using 20 different mobile phones of different make and model such as SAMSUNG on 7 pro, VIVO Y211, SAMSUNG note 8, REDMI note 8 A, REDMI Note 5, REALME model CPH 1859, ONEPLUS 6T, MOTOROLA, MI A3 model, REDMI k 20. Some video samples were also taken from the actual crime cases received from different investigation agencies from throughout India.

\subsection{Video Editing Software used}

More than 12 video editing software were used to make the video samples for analysis purpose. six (07) executive software i.e Corel Video Studio 2018, Video Pad Video Editor, Wondershare Filmora, AVS Video Editor, Forevid, Shotcut version and Advanced Video Cutter, two (03) online video editors i.e. YOUCOT pro, VideoGur and two more than mobile-based video editor i.e. video cutter, clipchamp.com/editor online \& Clipchamp.com/editor online software were used for editing the video samples for analysis purpose.

\subsection{The Software used for extraction and analysis of metadata and File containers (atoms/boxes)}

The MediaInfo, MediaTab, METADATA 2GO.com Exif info, Moo0 Video Info, Movie Scanner freeware softwares were used to extract the metadata information from original and edited MP4 video samples. The MediaInfo has a different view but (HTML \& XML) which may be utilized to view the metadata. In the case of MediaTab two option are available i.e. basic and advance, both can be used as per requirement. The details of the metadata information used for analysis are mentioned in Table.2. The profile format, device info, codec details, writing program, GOP details, frame rate mode of video, encoded library details are the main artefacts which used for identification of original and edited video.

For the extraction of MP4 file containers (boxes/atoms) and the analysis of their content the "MP4 inspector, mp4-explorer and Online Mp4 Parser-www.onlinemp4parser.com, were used. The MP4 video format contained more than 46 atoms as mentioned above in the table.4 Fig.1a \& Fig 1b. Every atom has unique information. But in our purposes the atoms and their contents are useful may be mentioned as "ftyp", "moov", "mvhd", Video

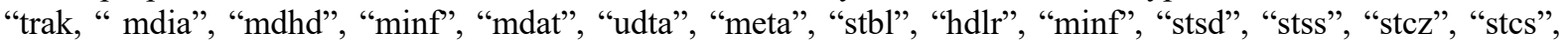
"ttds.

\section{RESULT AND DISCUSSION}

\subsection{Study of Metadata of the MP4 Video files}

The MP4 video file store metadata information as per ISS/IEC 14496-12:2005(E). This information almost are common in all mobile phone MP4 recordings. Whenever the original video recording edited using any video editor the various metadata properties will change depends upon the codec used. We have analyzed more than 200 original and edited video files for their metadata information. In one example the video pad editor software was used to edit the video and their metadata were compared as shown in Table.2. In the edited file, the format profile changed "Base Media/version.2 to "Base Media", File Codec ID changed from mp42(isom/mp42) to isom(isom/iso2/Avc1/mp41). The UTC details and device info is missing in the edited file. The New writing application and writing library with encoding setting appear in the edited video. The GOP (group of picture), mvhd(media header info) are missing. The Frame rate mode in original which is variable becomes constant in the edited video.

We have analyzed more than 200 video samples. The original and edited video(made by different video editor) were analyzed for their metadata information. The observations/ artifacts which are different from the original file are as mentioned in details. The common observations for all cases, it was found that the date of creation (DOC), date of modification (DOM), UTC date and time, changed in the edited video. The recording device info i.e android version/make, GOP information, mdhd (media header) are missing in the edited video. The frame rate mode of the video change variable to constant. Most of the cases the edited video contained the details of writing program/application used in editing. This is very crucial evidence showing the file has been edited. The H.264 is the very common writing library/Codec used in editing software. 
The Lavf is an audio/video library containing demuxers and muxers widely used by most of the video editors. However, their version may be different from editor to editors. It is Lavf 55.52.102 for video pad editor, Lavf 58.29.100 for hotcut, Lavf55.33.100 for Advanced Video Cutter, Lavf58.35.101 for Video Cut(mobile) etc. The x264 is the common encoder used MP4 format or other compression formats. Its version is different such as in VPE it is x264 core 142 while x264 core 114 for Forvid, x264 core 148 for Video guru etc. The format profile for MP4 format is High@L4 but in case of the edited video, it may be changed Simple@L1(forVPE), High@L3.2 (Advance Video Cutter) etc. For Corel VideoStudio 2018 the format Codec Id Changed mp42 (isom/mp42) to isom (mp41/mp42 and format profile changed High@L4 to Main@L4. In case of audio metadata only minor changed were observed i.e UTC details missing. The details of observations of 11 video edited by different video editors is shown in Table.3

\subsection{Study of File containers (boxes/atoms)}

The MP4 Container is mainly specified by ISO/IEC 14496-12, although some boxes are elaborated by ISO/IEC 14496-15 and ISO/IEC 14496-14. Hence, the MP4 format has the defined boxes and they are in a fixed arrangement/order. The original MP4 video format contained three main boxes i.e. "ftyp", "mdata", "move". When the video file edited the number of boxes/atoms and their arrangement will also Change. Table. 4 (Fig.1a \& Fig. 1b). The original file contained 46 boxes while edited file having 52 boxes. The atom "ftyp" contained the information about file major brand and minor version. In original, the major brand is "mp42(MP4 v2" while in the edited file it changes "isom(MP4 Base Media V1". The minor brand changed 0 to 512 in the edited video. In one example video pad editor the changes are shown in Fig.2a \& Fig $2 b$ in table 4. The "moov" is the parental box contained "mvhd", "meta" and two "trak" boxes video and audio are existing in the original file. Whenever the file is edited the whole structure of boxes inside "moov" will be changed including its contents Fig3 \& Fig 3b. Such as in "mvhd" containing details about DOC, DOM and matrix, will be changed in the edited file. In the original file, the box "udta" contained the atoms "meta" and "@xyz" which contained the information about recording device information (may be contained device make and model) and GPS coordinates. While in the edited file the either whole "udta" box either missing or it comes the box "meta" shift in this box, The atom "meta" contained very important information i.e. in original make and version of recording device but in case of the edited video, it contained the details of writing program/ codec details in a newly created atoms (Ctoo as shown in Fig4a \& Fig.4b.

The video atom "trak" has very s significant information about the video file. Its atoms distribution is shown in Fig.5a \& Fig.5b. The atom "trak" contained mainly two-atom i.e. " tkhd" \& "mdia" but some make of mobile contained one extra atom edit box "edst" and its child atom "elst". The "tkhd" contained video track information UTC details and matrix structure. These information would change in edited file. The atom "mdia" contained three atoms i.e. " mdhd " (media header), " hdlr" (handler reference box) \& "minf" (media information atom). The "minf" contained three child atoms " vmhd", " dinf" \& " stbl" . The atom "stbl" sample table contained wide information including chunk details. The atom "stbl" may contained six or seven child atoms i.e "stsd"- sample description atom, "stts"- time to sample, "stsc"-sample to chunk, "stsz"-sample size, "stco"-chunk of set. In original MP4 video the atom "stsd" contained file avc1, which further contained further three atoms "avcc", "pasp", "colr"), while in edited video contained only one atom "avcc" in which video codec may be different. In atom "stts" the sample delta reduced significantly (5944 to 389). In size/entry/chunk of the atome "stts" (sample time), "stss"(sync sample box), "stsc"(sample to chunk box), "stsz" (sample size box), "stco" sample to chunk box but "ctts" (sample to composition box) is missing in the edited box. In audio "trak" one extra box "edts" comes in the edited file. In other boxes, only number of sample count reduced significantly

\subsection{Study of MP4 Video captured by mobile Video Camera app/Video Recorder Apps}

There are two types of camera apps, First having only video capturing option and other having capturing and editing both options. We have recorded many videos in MP4 format using apps such as Camera MX Ver.4.7.200, HD video Rec0rder, Wonder Video, FILMIX Ver. 2.3.2, Filmigo Ver.4.7.6, AndroVid Ver.4.1.4, camera ver. 1.6.0. The recorded videos were analyzed for their media information and file containers. In case of simple video recording camera apps, the almost the all metadata properties remain unchanged only the "udta" which contains the information about GPS is missing. The structural change in atoms some changed were observed. The atom "moov" shift in last in case of video recorded by the app. The details are shown in Fig 6a \& Fig.6b 
Table.1 (Metadata information of the MP4 video)

\begin{tabular}{|c|c|c|c|c|c|}
\hline & \multirow[b]{2}{*}{\begin{tabular}{|l|} 
Video \\
ID: \\
Format $:$ \\
Format/Info : \\
Format profile : \\
Format settings : \\
Format settings, CABA \\
Format settings, Refere \\
Format settings, GOP
\end{tabular}} & & \\
\hline $\begin{array}{l}\text { Complete name: } \\
\text { Format: } \\
\text { Format profile: } \\
\text { Codec D : } \\
\text { File size : } \\
\text { Duration : } \\
\text { Overall bit rate : } \\
\text { Encoded date : } \\
\text { Tagged date : } \\
\text { xyz: } \\
\text { com.android.version: }\end{array}$ & 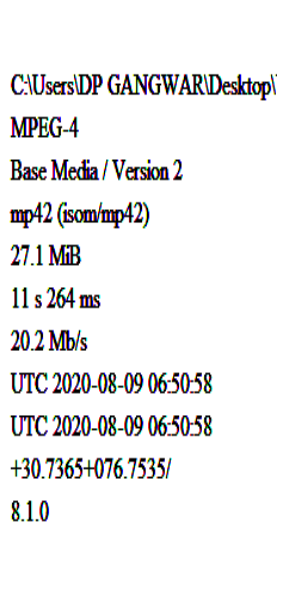 & & $\begin{array}{l}1 \\
\text { AVC } \\
\text { Advanced Video Codec } \\
\text { Baseline@L4 } \\
1 \text { Ref Frames } \\
\text { No } \\
1 \text { frame } \\
\mathrm{M}=1, \mathrm{~N}=30 \\
\text { avc1 } \\
\text { Advanced Video Coding } \\
11 \mathrm{~s} 252 \mathrm{~ms} \\
19.8 \mathrm{Mb} / \mathrm{s} \\
1920 \text { pixels } \\
1080 \text { pixels }\end{array}$ & \begin{tabular}{|l} 
Audio \\
D : \\
Format : \\
Format/Info: \\
Codec DD: \\
Duration : \\
Bit rate mode : \\
Bit rate : \\
Channel(s) : \\
Channel layout : \\
Sampling rate : \\
Frame rate : \\
Compression mode : \\
Stream size : \\
Title : \\
Language : \\
Encoded date : \\
Tagged date : \\
mdhd_Duration:
\end{tabular} & $\begin{array}{l}2 \\
\text { AAC LC } \\
\text { Advanced Audio Codec Low Complexity } \\
\text { mp4a-40-2 } \\
11 \text { s } 264 \mathrm{~ms} \\
\text { Constant } \\
96.0 \mathrm{~kb} / \mathrm{s} \\
2 \text { channels } \\
\text { L R } \\
48.0 \mathrm{kHz} \\
46.875 \mathrm{FPS}(1024 \mathrm{SPF}) \\
\text { Lossy } \\
132 \mathrm{KiB}(0 \%) \\
\text { SoundHandle } \\
\text { English } \\
\text { UTC 2020-08-09 06-50-58 } \\
\text { UTC 2020-08-09 06-50-58 } \\
11264 \\
\end{array}$ \\
\hline
\end{tabular}

\begin{tabular}{|c|c|c|}
\hline 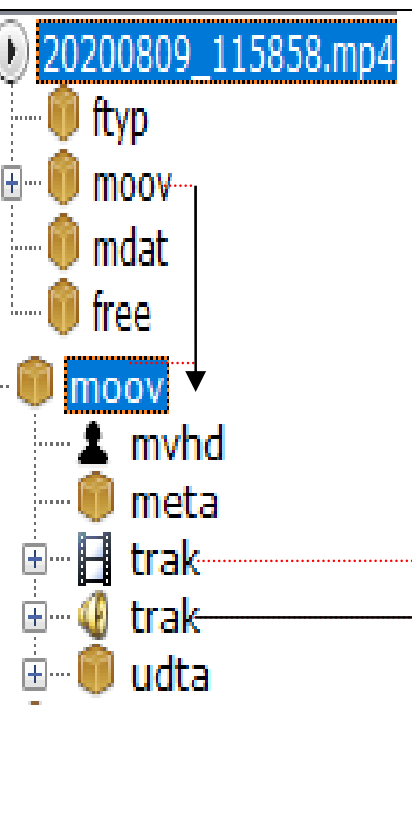 & 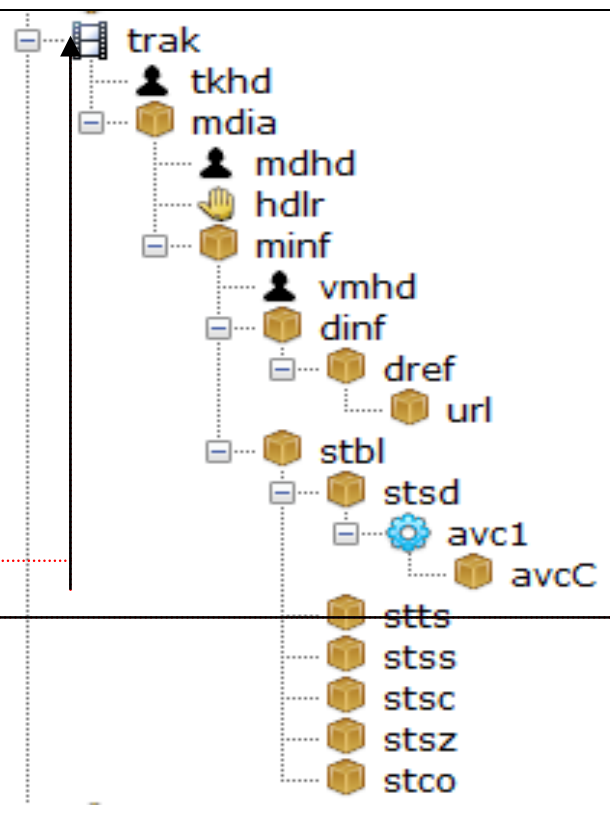 & 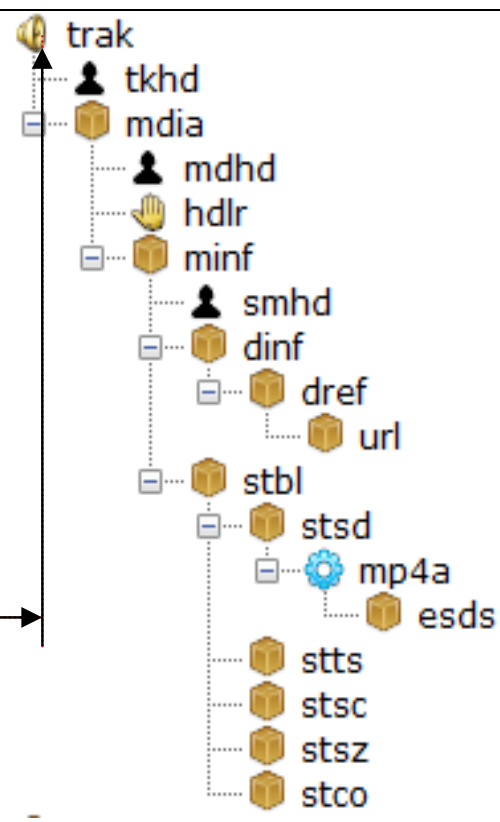 \\
\hline
\end{tabular}

Figure. (1a) Structure of MP4 box

Figure. (1b) Video "trak" atom

Figure. (1c) Audio "trak" atom 
Table.2 (Comparison of the metadata information between the original and edited files)

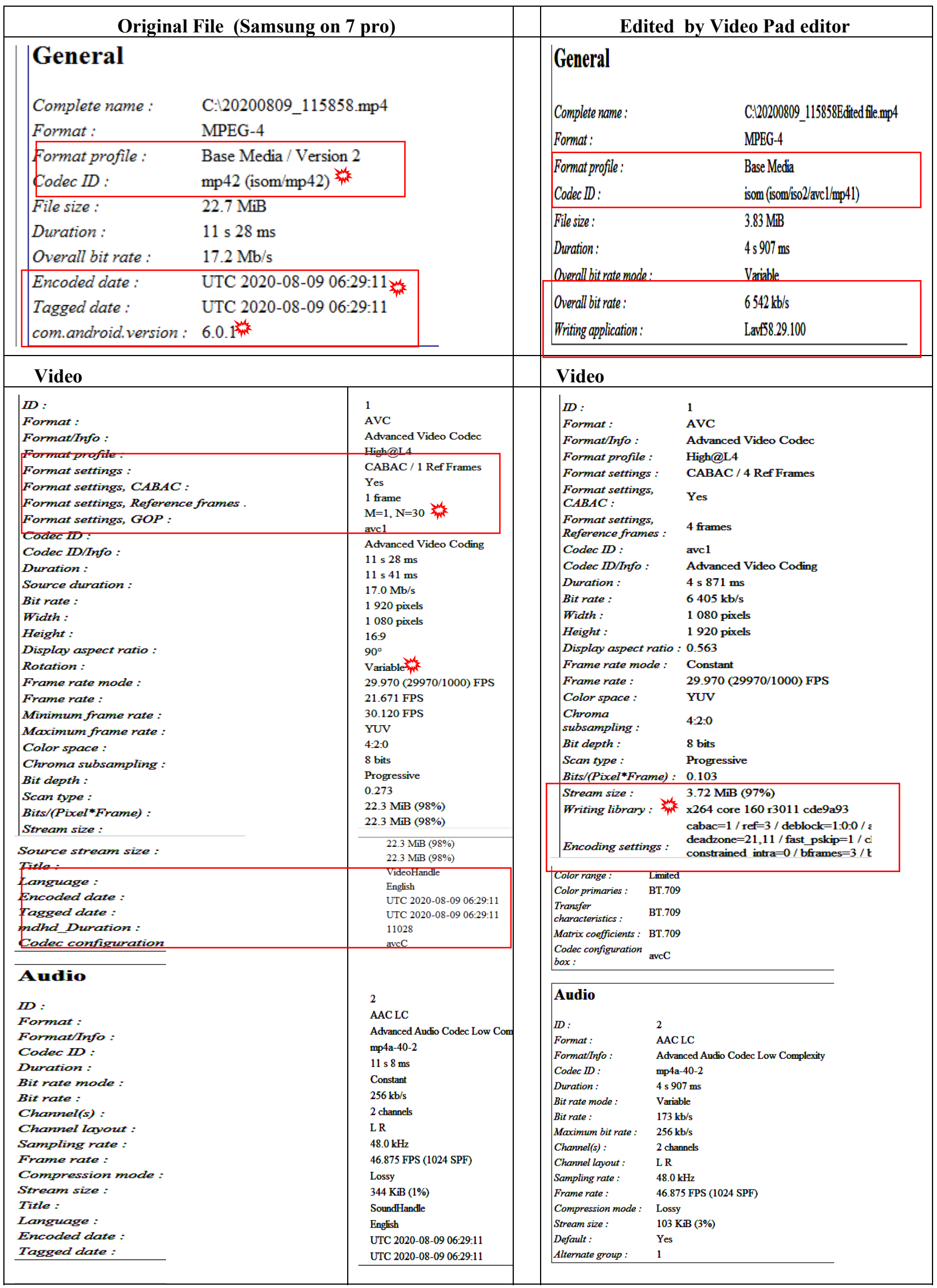


Table. 3: Artifacts observed in the metadata in different edited mp4 videos

\begin{tabular}{|c|c|c|}
\hline S.N. & Video Editor used & Artifacts found in Metadata of editing video \\
\hline 1 & $\begin{array}{l}\text { Common changed } \\
\text { occurred in the edited } \\
\text { video }\end{array}$ & $\begin{array}{l}\text { i. The date of creation (DOC), date of modification (DOM), UTC } \\
\text { date and time, changed in the edited video. } \\
\text { ii. The recording device info i.e android version/make etc. delete in } \\
\text { the edited video. } \\
\text { iii. The GOP information missing in the edited video. } \\
\text { iv. The frame rate mode of the video changes variable to constant. } \\
\text { v. "mdhd" missing. }\end{array}$ \\
\hline 2 & Corel Video Studio 2018 & $\begin{array}{l}\text { Codec ID : Changed: mp42 (isom/mp42) to isom (mp41/mp42), format } \\
\text { profile High@L4 to Main@L4. }\end{array}$ \\
\hline 3 & VideoPad Video Editor & $\begin{array}{l}\text { Writing application:Lavf55.33.100, Writing library: Lavc55.52.102 } \\
\text { with Encoding settings present, Codec ID: changed with 41, Video } \\
\text { format: Change avc to MPEG-4 Visual, Codec ID: changed from ave1 } \\
\text { to MPEG-4 Visual, }\end{array}$ \\
\hline 4 & Wondershare Filmora9 & $\begin{array}{l}\text { Codirector: Codirector, Production studio: Studio, Codec ID : } \\
\text { Changed (mp42 (isom/mp42 to isom (isom/iso } 2 / \mathrm{avc} 1 / \mathrm{mp} 41) \text {. }\end{array}$ \\
\hline 5 & AVS Video Editor 9.4 & Writing library: AVS, File Codec ID: changed \\
\hline 6 & Forevid & $\begin{array}{l}\text { Format profile: Changed Base Media / Version } 2 \text { to JVT, Codec ID : } \\
\text { Changed mp42 (isom/mp42) to avc1 (isom/avc1), Writing library: x } 264 \\
\text { core } 114 \text { with Encoding settings present. }\end{array}$ \\
\hline 7 & Shotcut version 20.07.11 & $\begin{array}{l}\text { File Codec ID: Changed (mp42 (isom } / \mathrm{mp} 42 \text { to isom } \\
\text { (isom/iso2/avc1/mp41) } \\
\text { Writing application: Lavf58.29.100, Writing library: x264 core } 160 \\
\text { r3011 cde9a93 with Encoding settings present. }\end{array}$ \\
\hline 8 & Advanced Video Cutter & $\begin{array}{l}\text { Writing application :Lavf55.33.100, Writing library: } \mathrm{x} 264 \text { core } 148 \\
\text { with Encoding settings present, File Codec ID : (mp42 (isom/mp42 to } \\
\text { isom (isom/iso } 2 / \mathrm{avc} 1 / \mathrm{mp} 41) \text {. }\end{array}$ \\
\hline 9 & YOUCOT pro (Mobile) & $\begin{array}{l}\text { Writing application: Lavf57.83.100, File Codec ID : (mp42 } \\
\text { (isom/mp42 to isom (isom/iso2/avc1/mp41), Title: ideoHandle,: } \\
\text { missing, Format settings CABAC: Change yes to NO }\end{array}$ \\
\hline 10 & Video Cut (mobile) & $\begin{array}{l}\text { Writing application: Lavf58.35.101, File Codec ID : (mp42 } \\
\text { (isom/mp42 to isom (isom/iso2/avc1/mp41), Title: ideoHandle,: } \\
\text { missing, Format settings CABAC: Change yes to NO }\end{array}$ \\
\hline 11 & VideoGure (Mobile) & $\begin{array}{l}\text { Video Format profile: High@L4 to Baseline@L5.2, Writing } \\
\text { application : Lavf57.83.100, Writing library: x264 core } 148 \text { with } \\
\text { Encoding settings present, File Codec ID : (mp42 (isom/mp42 to isom } \\
\text { (isom/iso2/avc1/mp41), GOP Missing, Title: VideoHandle: missing, } \\
\text { Format settings CABAC : Change yes to No. }\end{array}$ \\
\hline 12 & $\begin{array}{l}\text { clipchamp.com/editor } \\
\text { online }\end{array}$ & $\begin{array}{l}\text { Video Format profile: High@L4 to Baseline@L4.1, Writing } \\
\text { application: https://clipchamp.com, Writing library: x264 core } 158 \text { with } \\
\text { Encoding settings present, File Codec ID: changed (mp42 (isom/mp42 } \\
\text { to isom (isom/iso2/avc1/mp41), GOP Missing, Title: missing, Format } \\
\text { settings CABAC: Change yes to No. }\end{array}$ \\
\hline 13 & https://www.wevideo.com & $\begin{array}{l}\text { Writing application : Lavf58.45.100, Writing library: } \mathrm{x} 264 \text { core } 152 \\
\text { r2854 e9a5903 with Encoding settings present, File Codec ID : (mp42 } \\
\text { (isom/mp42 to isom (isom/iso2/avc1/mp41), Title: missing, Format } \\
\text { settings CABAC: Change yes to No. }\end{array}$ \\
\hline
\end{tabular}


Table:.4 (Comparison of the atoms details between original file and edited file)

\begin{tabular}{|c|c|c|c|c|c|}
\hline $\begin{array}{l}\text { (1) 20200809_115858.mp4 } \\
\text { ftyp } \\
\text { mdat } \\
\text { moov }\end{array}$ & $\begin{array}{l}\text { Item } \\
\text { File Information } \\
\text { Size } \\
\text { Cath } \\
\text { Creation Time } \\
\text { Last Write Time } \\
\text { Lildren Boxes } \\
\text { Last Access Time } \\
\text { Ttyp } \\
\text { Total Boxes } \\
\text { moov }\end{array}$ & 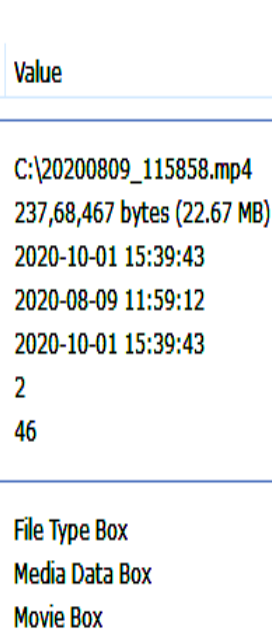 & $\begin{array}{l}\text { DPE Untitled.mp4 } \\
\text { ftyp } \\
\text { moov } \\
\text { free } \\
\text { mdat }\end{array}$ & 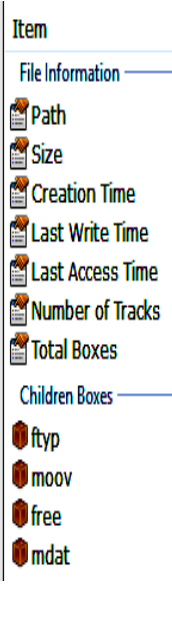 & 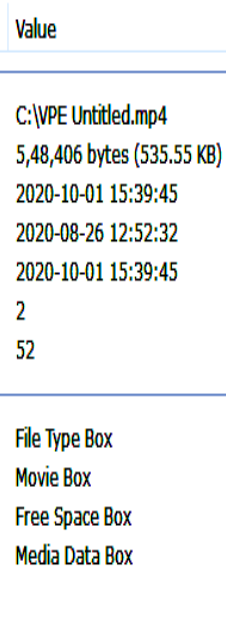 \\
\hline
\end{tabular}

Figure.2a(Original Video File)

Figure.2b(Edited Video File

\begin{tabular}{|c|c|c|c|}
\hline \multicolumn{2}{|l|}{ Box Data } & \multirow{2}{*}{$\begin{array}{l}\text { Eajor Brand } \\
\text { Minor Version }\end{array}$} & \multirow{2}{*}{$\begin{array}{l}\text { isom (MP4 Base Media v1) } \\
512\end{array}$} \\
\hline Major Brand & mp42 (MP4 v2) & & \\
\hline Minor Version & 0 & & \\
\hline \multicolumn{2}{|l|}{$<$} & \multicolumn{2}{|l|}{$<<$} \\
\hline & & \multirow{4}{*}{$\begin{array}{l}\text { Compatible Brand } \\
\text { :isom } \\
\text { :iso2 } \\
\text { : } \text { imp41 }\end{array}$} & Description \\
\hline Compatible Brand & Description & & MP4 Base Media v1 \\
\hline ":isom & MP4 Base Media v1 & & MP4 Base Media v2 \\
\hline :mp42 & MP4 v2 & & MP4 v1 \\
\hline
\end{tabular}

Figure.3a (original File)

Figure. 3(edited file)

(Difference between major brand, minor version, compatible brand)

\begin{tabular}{|c|c|}
\hline 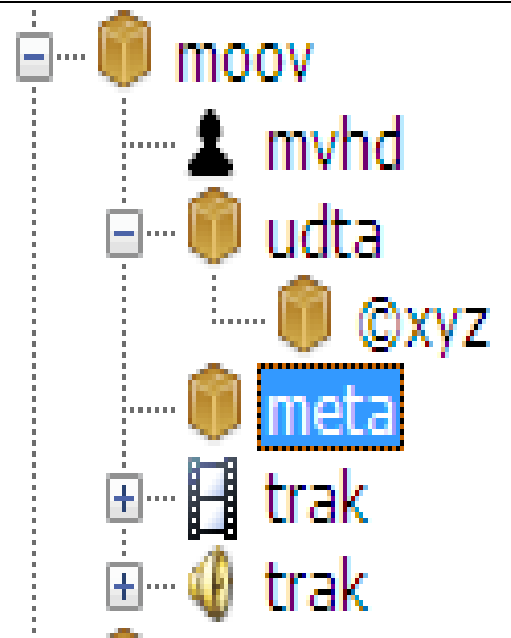 & 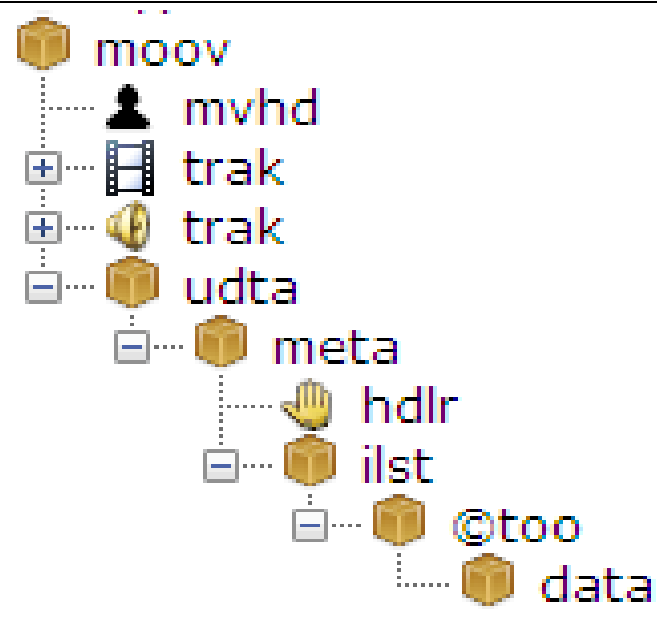 \\
\hline
\end{tabular}

Figure.4a (original File)

Fig.3b(edited file)

(Details about rearrangement of atoms and children atom of "moov" atom ) 


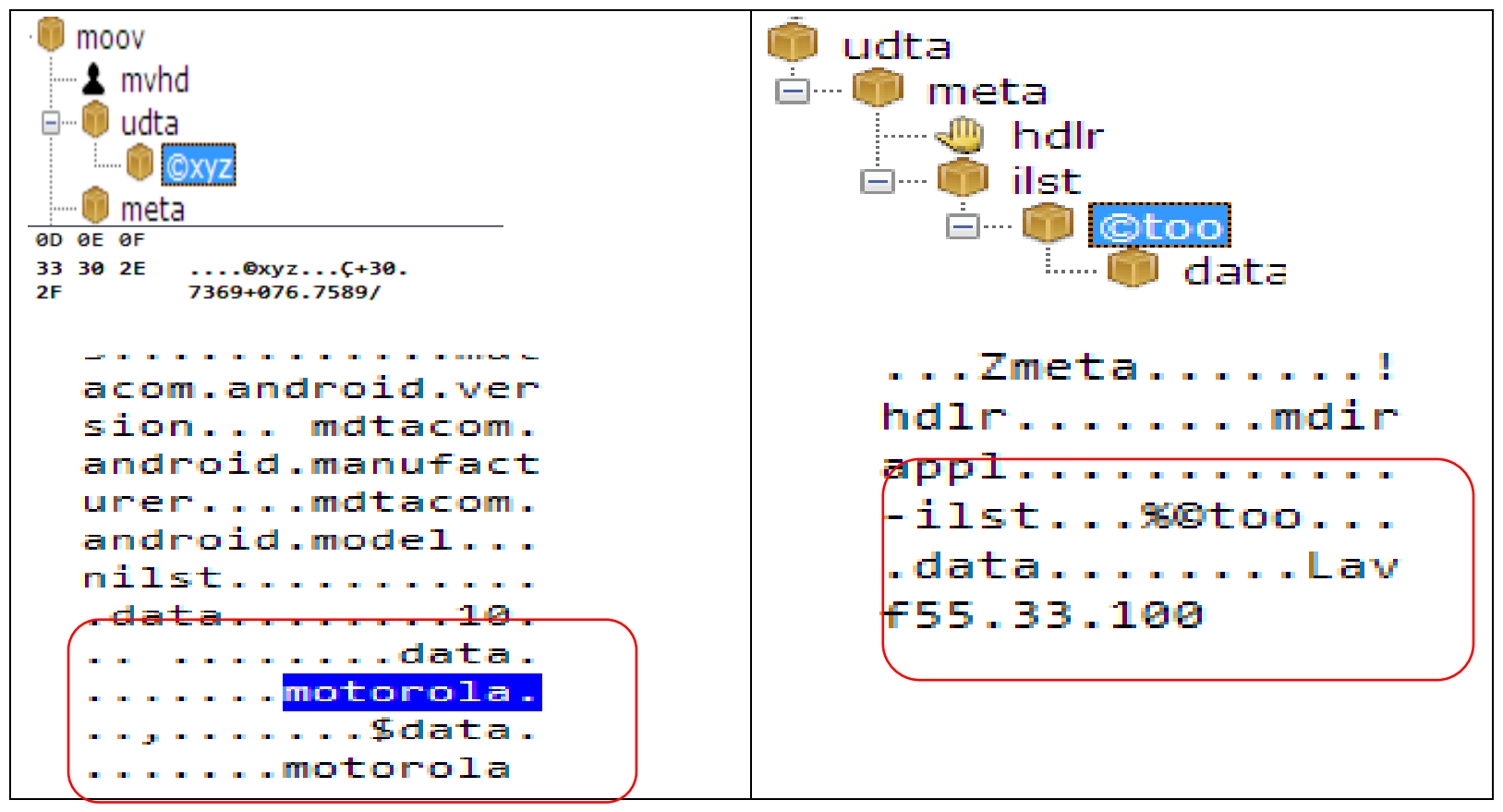

Figure.5a (original File)

Figure.5b(edited file)

(Difference between contents of atom "udta", "meta" and its contents )

\begin{tabular}{|c|c|}
\hline 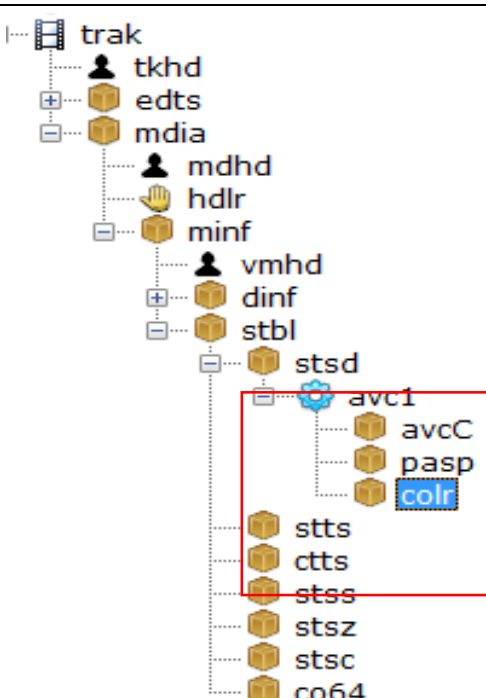 & 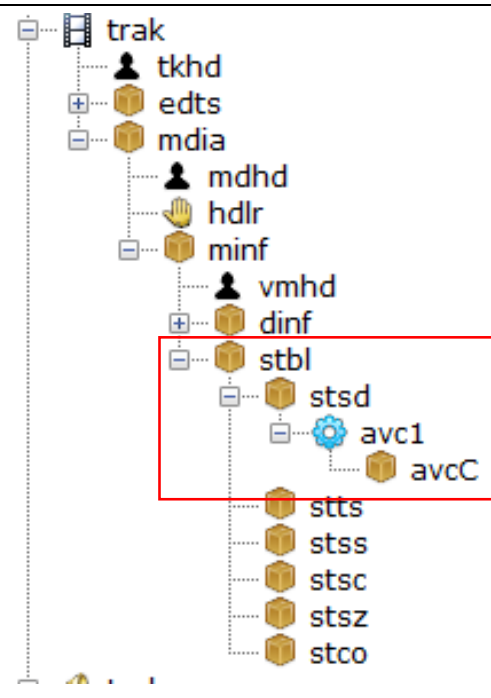 \\
\hline
\end{tabular}

Figure.6a (original File)

Figure.6b(edited file)

(Arrangement of atom "trak" Video in original and edited file ) 


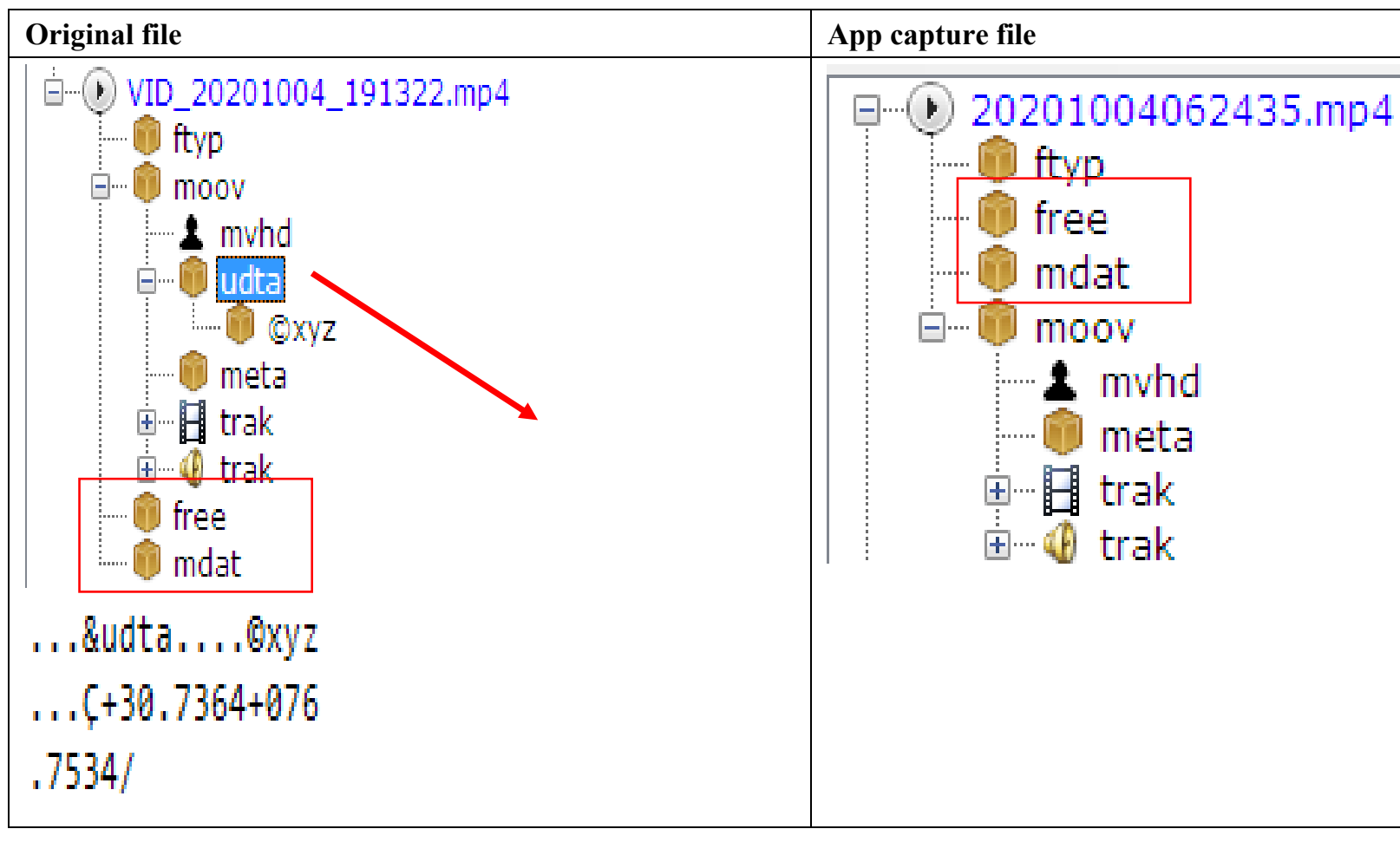

Figure.7a

Figure.7b.

\section{CONCLUSION}

The findings of our research of the analysis of original and edited digital video reveals that when the video was edited using any type the video editor it leaves its own sign(s) in form of metadata properties as well as of rearrangement of boxes/atoms of file containers and in its contents also. The artifacts identified in the edited video as described above in details provide valuable clues to verify the authenticity of MP4 video files. The proposed method can be applied in addition with conventional method of audio/video analysis authentication of MP4 video recordings for fast and better accuracy.

\section{Future work}

The present study is limited only for MP4 video. Hence, it is felt that more research is required to cover other video formats like AVI, MOV, 3GP, WMV, MKV and other video formats. Future research will be carried out subsequently.

\section{ACKNOWLEDGMENTS}

The author is thankful to Dr. S. K Jain, Director of Central Forensic Science Laboratory, Chandigarh for his guidance. The author is also very thankful to Dr. Navin Aggarwal Prof. UIET, Punjab University, Chandigarh for his valuable suggestions.

\section{REFERENCE}

[1] D.P. Gangwar, Anju Pathania " Authentication of Digital Image using metadata and decoding Properties" IJSRCSEIT, Volume 3 Issue 8 page $2456-3307$.

[2] D.P. Gangwar, Anju Pathania " Authentication of Digital Audio Recording using file signature and metadata Properties" IJEAST, Volume 5 Issue 3 page $162-165$.

[3] Martin Harran, William Farrelly, Kevin Curran "A Method for Verifying Integrity \& Authenticating Digital Media"Applied Computing and Informatics (2017), doi: http://dx.doi.org/10.1016/j.aci.2017.05.006.

[4] Thomas Gloe, André Fischer, Matthias Kirchner "Forensic Analysis of Video Format" Digital Investigation 11 (2014) S68-S76" www.elsevier.com/locate/diin.

[5] J. Randolph hall "mpeg--4 video authentication using file structure and metadata" B.F.A., Ithaca college, 2002 a thesis of masters of science 2015 .

[6] Jieun Song, Kiryong Lee, Wan Yeon Lee, Heejo Lee “ Integrity verification of the ordered data structure in manipulated video contents" Digital Investigation 18 (2016) 1-7": www.elsevier.com/locate/diin.

[7] David Gijeera, Sriram Baireddy, Paolo Bestagini, Stefano Tubaro, Edward J. Delp, "We Need No Pixels: Video Manipulation Detection Using Stream Descriptors" arXiv:1906.08743v1 [cs.LG] 20 Jun 2019.

[8] RAQUEL RAMOS LÓPEZ, ELENA ALMARAZ LUENGO, ANA LUCILA SANDOVAL OROZCO, 1, AND LUIS JAVIER GARCIA VILLALB "Digital Video Source Identification Based on Container's Structure Analysis" Received December 19, 2019, accepted January 6, 2020, date of publication February 5, 2020, date of current version March 2, 2020 Digital Object Identifier 10.1109/ACCESS.2020.2971785.

[9] BRIAN C. HOSLER and others, "The Video Authentication and Camera Identification Database: A New Database for Video Forensics" Received April 30, 2019, accepted May 29, 2019, date of publication June 10, 2019, date of current version June 26, 2019 
Digital Object Identifier 10.1109/ACCESS.2019.2922145.

[10] Dasara Shullani and others: a video and image dataset for source identification" EURASIP Journal on Information Security (2017) 2017:15. DOI 10.1186/s13635-017-0067-2.

[11] Massimo Iuliani, Dasara Shullani, Marco Fontani, Saverio Meucci, and Alessandro Piva "A Video Forensic Framework for the Unsupervised Analysis of MP4-like File Container" IEEE TRANSACTIONS ON INFORMATION FORENSICS AND SECURITY, DOI 10.1109/TIFS.2018.2859760, IEEE.

[12] Carlos Quinto Huamán, Ana Lucila Sandoval Orozco and Luis Javier García Villalba "Authentication and Integrity of Smart Phone Videos through Multimedia container structure analysis” Future Generation Computer Systems 108 (2020) 15-33, journal homepage: www.elsevier.com/locate/fgcs.

[13] Apple, "Introduction to quicktime file format specification,"2015. 